\title{
Clustering and heterogeneous dynamics in a kinetic Monte-Carlo model of self-propelled hard disks
}

\author{
Demian Levis and Ludovic Berthier \\ Laboratoire Charles Coulomb, UMR 5221 CNRS and Université Montpellier 2, Montpellier, France
}

(Dated: September 25, 2018)

\begin{abstract}
We introduce a kinetic Monte-Carlo model for self-propelled hard disks to capture with minimal ingredients the interplay between thermal fluctuations, excluded volume and self-propulsion in large assemblies of active particles. We analyze in detail the resulting (density, self-propulsion) nonequilibrium phase diagram over a broad range of parameters. We find that purely repulsive hard disks spontaneously aggregate into fractal clusters as self-propulsion is increased, and rationalize the evolution of the average cluster size by developing a kinetic model of reversible aggregation. As density is increased, the nonequilibrium clusters percolate to form a ramified structure reminiscent of a physical gel. We show that the addition of a finite amount of noise is needed to trigger a nonequilibrium phase separation, showing that demixing in active Brownian particles results from a delicate balance between noise, interparticle interactions and self-propulsion. We show that self-propulsion has a profound influence on the dynamics of the active fluid. We find that the diffusion constant has a nonmonotonic behaviour as self-propulsion is increased at finite density and that activity produces strong deviations from Fickian diffusion that persist over large time scales and length scales, suggesting that systems of active particles generically behave as dynamically heterogeneous systems.
\end{abstract}

PACS numbers: 05.10.Ln, 47.57.eb, 82.70.Dd

\section{INTRODUCTION}

In many active materials, the relevant entities (molecules, cells, animals...) are self-propelled objects which can borrow energy from their environment to produce their own motion using some internal mechanism [13. At the fundamental level, the local energy input required by self-propulsion breaks detailed balance, which automatically drives the system far from thermodynamic equilibrium. Self-propulsion is therefore directly responsible for the emergence of a number of collective phenomena that are not observed in the absence of activity, such as, for instance, the existence of an orientationally ordered state in two spatial dimensions [1 4, the formation of coherently moving patterns, aggregation, clustering, motility-induced phase separation and giant number fluctuations [1-10.

Self-propelled 'particle' systems are manifold in biology, with examples ranging from animal groups to bacterial colonies or molecular motors in the cytoskeleton [2]. Recently, artificial or 'abiotic' physical systems of selfpropelled particles have been realised in the laboratory using, for instance, granular materials [1, 12, or colloidal particles with specific surface treatments 13 19. These experimental developments offer an ideal playground to investigate general features of self-propelled particle systems since they are simpler to control and more versatile than their biological counterparts. Because the physics of granular and colloidal particles has been analyzed in great detail, self-propulsion can be seen as a new physical ingredient whose influence needs to be studied. In this work, our central objective is to understand how deviations from detailed balance originating from persistent self-propulsion affects the structure and dynamics of simple fluids.
Over the last few years, the field of active matter has been the subject of a large number of studies, striking interest from a broad community of researchers stemming from different fields $[1]$. Previous studies have revealed the importance of the shape and polarity of selfpropelled particles for their collective behaviour. Agentbased models of polar particles with an alignment interaction undergo a phase transition towards a polar ordered state, resulting in giant number fluctuations [4, 6, 20. Elongated apolar self-propelled particles such as granular rods or myxobacteria with excluded volume interactions order in a nematic state with no polar collective motion. Moreover, nematic particles self-organise into coherently moving patterns [5, 11, 21]. Closer to simple liquids and to our own approach, recent studies have shown that systems made of apolar isotropic particles with no orientational order may exhibit a motility-induced phase separation [7, 9, 22], which has motivated a large number of studies [23 28].

The recent development of simple experimental systems of self-propelled granular and colloidal particles motivates the study of simple model systems where the influence of self-propulsion on the equilibrium structure and dynamics of simple fluids can be understood in detail. The hard sphere model is perhaps the simplest and most studied model to study the physics of simple liquids [29], where physics stems from the competition between hard core repulsion and thermal fluctuations. Hard spheres represent also an excellent model to understand the physics of colloidal and granular particles. Therefore, we decided to develop a simple model of self-propelled hard particles, specializing ourselves to two dimensions where the majority of experiments on active particles are performed, but the model is easily generalized to treat higher dimensions. 
So far, the statistical mechanics of active particles has been studied numerically using mainly molecular dynamics simulations, where self-propulsion is introduced in Langevin descriptions of active Brownian dynamics by considering specific couplings between translational and orientational motion [8, 9, 22, 30, 31. In these descriptions, more complicated phenomena induced by additional coupling to hydrodynamic fluctuations are neglected, but they still contain an appreciable number of control parameters that must be simultaneously adjusted. Here, we wish to develop a minimal strategy to capture at the most basic level how self-propulsion affects the structure and dynamics of simple fluids, extending the approach successfully used to study simple fluids at thermal equilibrium, namely, kinetic Monte-Carlo simulations [32, 33. To our knowledge, Monte-Carlo simulations of active particle systems have not been reported so far. We will therefore carefully explain our modelling, justify its relevance for the field of active matter and compare it to alternative models in some detail.

The principal aim of our work is therefore to develop a 'minimal' numerical model with as few control parameters as possible. To this end, we introduce a kinetic Monte-Carlo model of self-propelled hard disk, where activity is controlled by a single control parameter, which reduces, in the dilute limit, to the persistence time $\tau$ of a persistent random walk motion. Equivalently, this control parameter can be seen as a 'rotational' Péclet number [34, 35. Because thermal fluctuations only affect rotational degrees of freedom, the 'translational' Péclet number is not a convenient control parameter in our model [9. The second control parameter of the model is the packing fraction $\phi$ of the hard disk system, which is the unique one in equilibrium conditions. Although our model is presumably too simple to describe the details of any specific experimental realization, we shall demonstrate that it can still capture some essential features of the interplay between thermal fluctuations, excluded volume, and self-propulsion.

The present work presents a detailed study of the $(\phi, \tau)$ phase diagram of the model, which reduces to the known equilibrium hard disk fluid model in the limit $\tau \rightarrow 0$. The glassy active dynamics of the model at large density is studied elsewhere [36], and we concentrate in this work on the regime $0 \leq \phi \leq 0.60$. Our main results are summarized in Fig. 11, which displays the emergence, at any finite density, of complex nonequilibrium structures in the active fluid, taking the form of finite size clusters at moderate density that percolate to form a gel-like structure at larger density. These structural changes are accompanied by profound changes in the dynamics of the system, which we also analyse in detail. Surprisingly, clustering in our model does not take the form of a macroscopic phase separation found in previous numerical models of active Brownian particles [7, 9, 22, 37, 38. We elucidate the physical origin of this qualitative difference by introducing an additional translational noise of strength $\eta$ in our model, and by showing that phase separation can

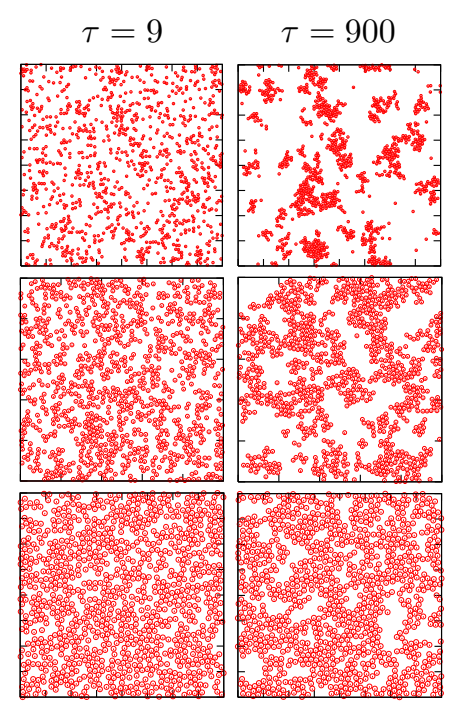

$\eta=0$ $\tau=900$

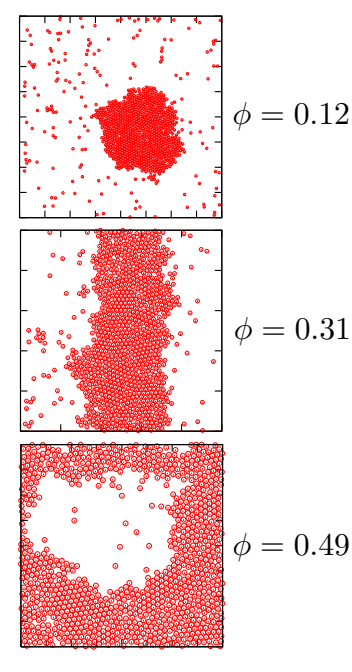

$\eta>\eta_{c}$
FIG. 1. Representative snapshots of a system composed of $N=10^{3}$ self-propelled hard disks at different packing fractions $\phi$. Left and central columns correspond to two different values of the persistence time $\tau$, showing a fluid phase at low $\phi$ and low $\tau$, the emergence of clusters as $\tau$ is increased, and a gel-like structure at large $\phi$ and large $\tau$. The right column shows phase separated systems obtained upon the addition of translational noise of finite amplitude $\eta$ across a broad range of densities.

only occur in a restricted part of the extended $(\phi, \tau, \eta)$ phase diagram where $\eta>0$ (see the corresponding snapshots in Fig. 1). This demonstrates that phase separation in fact results from a balance between noise, interparticle interactions and self-propulsion that is more delicate and perhaps less generic than previously thought. An interesting corollary is that clustering in our model can be interpreted as the result of a kinetically arrested phase separation, which adds a novel possible mechanism to the ongoing debate about the origin of clustering in selfpropelled particle systems [9, 17, 39, 40].

The paper is organised as follows. In Sec. II] we define our kinetic Monte-Carlo approach to simulate the behaviour of self-propelled hard disks, present the control parameters, units, behaviour in the dilute limit, and comparison with existing models. In Sec. III we establish the phase diagram of the model in steady state conditions, showing fluid, clustered and percolated phases whose boundaries are carefully studied. We construct and solve a kinetic model that reproduces the cluster size distribution obtained in the simulations and gives useful insights into the aggregation mechanism. In Sec. IV] we show that a finite amount of translational noise triggers a macroscopic phase separation over a broad range of densities. In Sec. V we analyse the dynamics of the model in steady state, and show that the relaxation of the system is highly heterogeneous. In Sec. VI we summarize and discuss our results. 


\section{KINETIC MONTE-CARLO MODEL FOR SELF-PROPELLED HARD DISKS}

\section{A. Hard disk model: Volume fraction $\phi$}

We work with a bidimensional, monodisperse assembly of $N$ hard disks of diameter $\sigma$ enclosed in a square box of linear size $L$, using periodic boundary conditions. The hard core interaction implies that no overlap between disk is allowed, whereas configurations with no overlap all have the same energy, which can be chosen to be zero, for convenience. Therefore, by contrast with most works dealing with active Brownian particles, the particle softness is not an independent control parameter, and density and temperature cannot be independently adjusted because we use an infinitely hard core repulsion. The system is thus uniquely characterized by the packing (or more precisely, area) fraction,

$$
\phi=\frac{\pi N \sigma^{2}}{4 L^{2}} .
$$

\section{B. Kinetic Monte-Carlo in equilibrium}

Monte-Carlo simulations are traditionally viewed as an efficient way to sample the configurational phase space with a given probability distribution, usually chosen as the Boltzmann distribution [32. However, it is well known that Monte-Carlo simulations can also be used to analyse the kinetics of statistical models, including off-lattice complex fluids [33].

For hard disks, the equilibrium Monte-Carlo approach is conceptually very simple, and proceeds as follows. The positions of the particles are updated sequentially, and dynamics results from the repetition of the following elementary Monte-Carlo trial step.

- At time $t$, a particle is chosen at random, say particle $i$.

- A random displacement $\vec{\delta}_{i}(t)$ is drawn from a chosen distribution. We shall use the following convention:

$$
\vec{\delta}_{i}(t)=\delta_{0} \vec{\xi}_{i}(t),
$$

where $\vec{\xi}_{i}(t)$ is a random vector with components independently drawn from a flat distribution in the interval $[-1,1]$, so that typical displacements have a typical amplitude $\approx \delta_{0}$.

- The move is accepted if it creates no overlap between the disks. This trial Monte-Carlo step can be written as

$$
\vec{r}_{i}(t+1)=\vec{r}_{i}(t)+\vec{\delta}_{i}(t) p_{\text {acc }}(t),
$$

where the acceptance probability $p_{\text {acc }}(t)$ is unity if no overlap is created, zero otherwise.
Conventionally, a Monte-Carlo time step $\tau_{\mathrm{MC}}$ is defined as the succession of $N$ such elementary moves, so that the dynamical behaviour expressed in units of $\tau_{\mathrm{MC}}$ does not depend on the number of particles in the limit of large $N$ 33.

Another interesting limit is when the elementary step size $\delta_{0}$ becomes very small. If this limit is considered, it is more convenient to use a different time unit, $\tau_{\mathrm{MC}}^{\prime}=$ $\sqrt{\delta_{0}} \tau_{\mathrm{MC}}$, such that the dynamics becomes independent of $\delta_{0}$ as $\delta_{0} \rightarrow 0$. In this limit, the above Monte-Carlo dynamics becomes equivalent to the following Brownian dynamics,

$$
\frac{\partial}{\partial t} \vec{r}_{i}(t)=\sum_{j} \vec{f}_{i j}(t)+\vec{\zeta}_{i}(t)
$$

where $\vec{f}_{i j}$ is the interparticle force, and $\vec{\zeta}_{i}$ a Gaussian random noise satisfying the fluctuation-dissipation theorem, $\left\langle\zeta_{i, \alpha}(t) \zeta_{j, \beta}\left(t^{\prime}\right)\right\rangle=2 \delta_{\alpha \beta} \delta_{i j} \delta\left(t-t^{\prime}\right)$.

\section{Introduction of self-propulsion: Persistence time $\tau$}

To introduce self-propulsion in the hard disk model, we must produce time correlations in the elementary particle displacements. A simple way to do this is to introduce correlations between successive displacements of the particles. To this end, we generalize Eqs. (2, 3) to

$$
\begin{aligned}
\vec{\delta}_{i}(t) & =\vec{\delta}_{i}(t-1)+\delta_{1} \vec{\xi}_{i}(t), \\
\vec{r}_{i}(t+1) & =\vec{r}_{i}(t)+\vec{\delta}_{i}(t) p_{\text {acc }}(t),
\end{aligned}
$$

where we enforce the condition that $\left|\delta_{i, \alpha}(t)\right| \leq \delta_{0}$, just as in the original equilibrium dynamics in Eq. (2).

The physics of the kinetic Monte-Carlo model in Eqs. (5. 6) is transparent, as it differs from the equilibrium Monte-Carlo dynamics only by the fact that the random displacement $\vec{\delta}_{i}(t)$ now performs a simple random walk of average jump length $\delta_{1}$ in a square of linear size $\delta_{0}$ (with reflective boundary conditions).

The interesting regime is when $\delta_{1}<\delta_{0}$, where the orientation of the elementary displacement then decorrelates slowly in about $\left(\delta_{0} / \delta_{1}\right)^{2}$ trial moves (this scaling stems from the fact that $\vec{\delta}_{i}$ must diffuse a distance $\delta_{0}$ using independent steps of size $\delta_{1}$ ). This implies that the elementary trial moves develop time correlations, and decorrelate after a typical persistent time $\tau$,

$$
\tau=\left(\frac{\delta_{0}}{\delta_{1}}\right)^{2} \tau_{\mathrm{MC}}
$$

which defines the second control parameter of the model. This timescale has sometimes been named rotational Péclet number [34, 35], as it quantifies the efficiency of the fluctuations that are responsible for the rotation of the displacement, as modelled by the term $\delta_{1} \vec{\xi}_{i}$ in Eq. (5). Note that it is not convenient to define a Péclet number 
based on translational degrees of freedom from Eq. (6), a situation encountered in previous models 9 .

The key feature of the kinetic model defined by Eqs. 5 6 is that whereas the model is Markovian in the enlarged space of positions and displacements, it becomes non-Markovian and time irreversible in the subspace of the positions, where detailed balance is therefore broken. As a result, the model becomes purely 'dynamical' in the sense that the configurational space of the hard disk system is not sampled according to the Boltzmann distribution anymore. In short, self-propulsion pushes the system far from equilibrium, which is only recovered in the limit $\tau \rightarrow 0$ where equilibrium sampling as in Eq. (2) is obtained. By slowly increasing $\tau$ we can then observe how the system departs from a well-known equilibrium situation. Such a smooth connection to equilibrium is not always possible in earlier models of active particles [9, 37.

In practive we have studied system sizes in the range $N=10^{3}-10^{4}$, to test against possible finite size effects which will be discussed whenever they are relevant. We have changed the persistence time $\tau$ in Eq. (7) using a fixed length scale $\delta_{0}=0.1$ controlling the maximal size of the elementary moves, and by varying $\delta_{1}$ in Eq. (5). We have explored a regime $\delta_{1}=0.001-0.1$, thereby covering a range of persistent times of 4 orders of magnitude, $\tau / \tau_{\mathrm{MC}}=1-10^{4}$.

From now on, we use the particle diameter $\sigma$ as the unit length scale, and the Monte-Carlo time step $\tau_{\mathrm{MC}}$ as the unit time scale.

\section{Dilute limit: persistent random walk}

The physics of the dilute limit is straightforward. The regime where $\tau \ll 1$ is trivial since particles then perform a simple random walk, with a jump size controlled by $\delta_{0}$, in which case, the mean-squared displacement defined as

$$
\Delta^{2}(t)=\left\langle\left[\vec{r}_{i}(t)-\vec{r}_{i}(0)\right]^{2}\right\rangle
$$

increases as $\Delta^{2}(t)=D_{0} t$, with $D_{0} \approx \delta_{0}^{2}$.

Let us consider the more interesting regime where $\tau \gg 1$. Two time regimes have then to be considered. When $t \ll \tau$, displacements are nearly persistent and the mean-squared displacement increases ballistically as $\Delta^{2}(t) \approx\left(\delta_{0} t\right)^{2}$. On the other hand, the displacements become uncorrelated for times $t \gg \tau$, and motion becomes diffusive, $\Delta^{2} \approx D t$, with

$$
D \approx D_{0} \tau
$$

This shows that, as expected in the dilute limit where particles do not interact, the self-propulsion mechanism enhances self-diffusion, simply because ballistic motion is more efficient than diffusion.

In Fig. 2, we present numerical data obtained from simulating the kinetic Monte-Carlo model Eqs. (5. 6) in the dilute limit, $\phi \rightarrow 0$. These data confirm the above description, with the observation of ballistic motion crossing over to diffusive behaviour at a time scale controlled

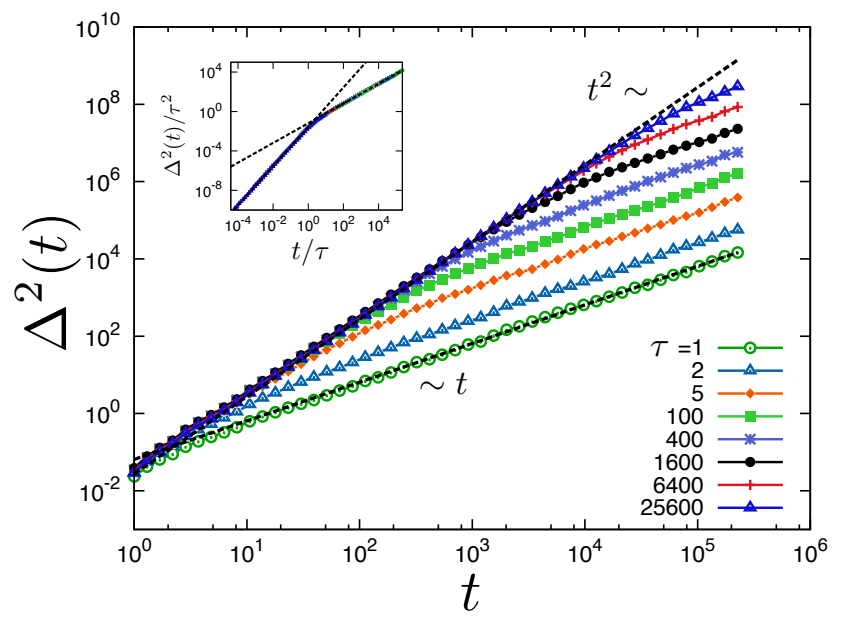

FIG. 2. Time dependence of the mean-squared displacement, Eq. (8), in the dilute limit, $\phi \rightarrow 0$, for different persistent times $\tau$, showing a crossover from ballistic to diffusive motion, with a diffusion constant that increases with $\tau$. Inset: The same data are collapsed using the scaling form in Eq. 10 with ballistic and diffusive asymptotics indicated with dashed lines.

by $\tau$, therefore reducing to simple diffusion in the equilibrium limit $\tau \rightarrow 0$. Because this crossover between ballistic and diffusive motion is uniquely controlled by the persistence time $\tau$, the mean-squared displacement takes the following scaling form,

$$
\Delta^{2}(t)=\tau^{2} F(t / \tau)
$$

where $F(x) \sim x^{2}$ for $x \ll 1$ and $F(x) \sim x$ for $x \gg 1$. This scaling form suggests that data can be collapsed when $\Delta^{2} / \tau^{2}$ is represented as a function of the rescaled time $t / \tau$, as demonstrated in the inset of Fig. 2. Physically, Eq. 10 also implies that in the dilute limit, it is equivalent to vary the persistence time or the velocity characterizing the ballistic motion at short time.

\section{E. Adding fluctuations: Translational noise $\eta$}

By definition of the kinetic model in Eqs. (5, 6), elementary particle displacements $\vec{r}_{i}(t+1)-\vec{r}_{i}(t)$ are fully slaved to the orientation of the vector $\vec{\delta}_{i}(t)$, whose time evolution is not affected by the particle motion. Thus, in our model, particles have only two choices: either move in the direction imposed by $\vec{\delta}_{i}(t)$ (when $p_{a c c}(t)=1$ ), or stop (when $p_{a c c}(t)=0$ ).

The main body of the article is devoted to studying the behaviour of the model using these simple kinetic rules. However, to establish connections with other models proposed for active Brownian particles [9, 22, 41, it will be useful to introduce an additional translational noise in our model. There are many possible ways to do this, and we have checked that our results do not depend crucially on the specific choice we made. 
We perturb the self-propelled dynamics in Eqs. (5, 6) by performing additional Monte-Carlo moves of small amplitude $\delta_{\eta}$ that have the same Markovian properties as in equilibrium dynamics. In practice, for each MonteCarlo trial, we choose with probability $1 / 2$ to use the selfpropelled dynamics in Eqs. (5. 6), or to draw a random displacement $\delta_{\eta} \vec{\xi}_{i}(t)$ (see Eq. 22 ). This perturbation then allows particle displacements that are not slaved to the direction of $\vec{\delta}_{i}(t)$, and therefore it introduces noise in our kinetic Monte-Carlo model.

We quantify the strength of the noise by the quantity $\eta=\delta_{\eta} / \delta_{0}$, which compares the relative size of the MonteCarlo moves performed without memory (of amplitude $\delta_{\eta}$ ) to the persistent moves (of amplitude $\delta_{0}$ ). Our original kinetic Monte-Carlo model is recovered as $\eta \rightarrow 0$, while for finite $\eta$, the model then lives in an extended three-dimensional phase diagram $(\phi, \tau, \eta)$.

Our primary motivation to introduce $\eta$ as an additional control parameter is to make contact with earlier numerical results, as detailed below. In particular, we emphasize that $\tau$ and $\eta$ separately control the strength of the self-propulsion and that of the noise, which will allow us to disentangle the relative role they might play in the physics of active Brownian particles.

\section{F. Comparison with earlier models}

The kinetic rules defining our Monte-Carlo model in Eqs. (5. 6) fall in between two types of modelling that have been put forward to understand the physics of active particle systems.

Because dynamics proceeds in discrete time steps, our model bears similarities with approaches initiated by Vicsek and coworkers, which analyse the dynamics of point particles evolving under the influence of aligning interactions and noise [2]. Although several distinct versions of such models have been studied in the literature, we are not aware of any Vicsek-like numerical model system incorporating physical ingredients comparable to ours.

A different class of models is obtained starting from Langevin equations governing the time evolution of the position and orientation of the self-propelled particles [9, 22, 41. Because we can take the limit of small step sizes in the kinetic Monte-Carlo approach, we expect our model to also bear strong similarities with Langevin models neglecting inertia, hydrodynamic interactions, and particle anisotropy, as studied for instance in Refs. [9, 22, 31, 37, 42]. It is therefore interesting to compare more precisely our model to this family of earlier studies.

To proceed we must first discuss the small step size limit in our model, in analogy with the discussion of equilibrium dynamics in Sec. IIB. To analyse the limit $\delta_{0} \rightarrow 0$, it is useful to introduce, again, a distinct time unit, $\tau_{\mathrm{MC}}^{\prime \prime}=\delta_{0} \tau_{\mathrm{MC}}$. In these novel (double-primed) units, the persistence time becomes $\tau^{\prime \prime}=\delta_{0} \tau$, persistent motion at short times $t^{\prime \prime} \ll \tau^{\prime \prime}$ takes place with a velocity $v^{\prime \prime}=1$, while the long-time diffusion coefficient controlling the regime $t^{\prime \prime} \gg \tau^{\prime \prime}$ in the dilute limit becomes $D^{\prime \prime}=\tau^{\prime \prime}$. The limit $\delta_{0}$ can then safely be taken, the physics remaining unaffected by the chosen $\delta_{0}$ value, provided the persistence time $\tau^{\prime \prime}$ is kept fixed. In practical terms, this implies taking the limits $\delta_{0} \rightarrow 0$ and $\delta_{1} \rightarrow 0$, while keeping the ratio $\delta_{0}^{3} / \delta_{1}^{2}=\tau^{\prime \prime}$ constant.

This reasoning applies as long as $\delta_{0}$ is small compared to any other length scale in the problem. We have checked that the numerical results presented in this paper are not affected by the specific choice $\delta_{0}=0.1$ we made. This choice is dictated by the usual trade-off between having a very small $\delta_{0}$ to get closer to the continuous time limit, and the large jumps needed to make simulations more efficient 43. Of course, extremely large jumps with $\delta_{0} \gg \sigma$ would be unphysical, and would anyway be rejected with high probability in dense systems.

Because the system is now far from equilibrium, equivalence with a Langevin description is not obvious. Earlier Langevin models typically study the following dynamical equations:

$$
\begin{aligned}
\frac{\partial}{\partial t} \vec{r}_{i}(t) & =v_{0} \vec{n}_{i}(t)+\sum_{j} \vec{f}_{i j}(t)+\vec{\zeta}_{i}(t), \\
\frac{\partial}{\partial t} \theta_{i}(t) & =\zeta_{i}^{R}(t),
\end{aligned}
$$

where $v_{0}$ is the 'bare' self-propulsion velocity, $\vec{n}_{i}=$ $\left(\cos \theta_{i}, \sin \theta_{i}\right)$ a unit vector whose orientation $\theta_{i}$ independently evolves according to Eq. (12). Note that this set of equations introduces several control parameters, having two sources of noise, an energy scale from the interparticle forces, and a velocity governing self-propulsion, which typically implies that only specific combinations of these are studied.

It is obvious that, in the dilute limit, such a Langevin description coincides with our kinetic Monte-Carlo approach, yielding in particular a persistent random walk similar to the data shown in Fig. 2, with a persistent time controlled by the strength of the angular noise $\zeta_{i}^{R}(t)$ in Eq. 12.

At finite density, the orientation evolves freely, and the analogy between Eqs. (5) and (12) still holds. However, the situation is different for Eq. (11) when interparticle forces compete with self-propulsion and noise. While displacements in our model are fully slaved to the orientation, Eq. (6), this is not the case in Eq. (11) which allows displacements transverse to the direction imposed by $\vec{n}_{i}$ resulting from the competition between forces and noise. This difference is responsible for the fact that some of our results differ from earlier reports. This is why we introduced the translational noise term $\eta$, as discussed above in Sec. IIE, in order to induce motion in the direction transverse to that of the self-propulsion. We will demonstrate below that this term is crucially needed to trigger a macroscopic phase separation.

Recently, a noiseless version of the above Langevin equations 11,12 has been studied, where $\vec{\zeta}_{i}=\overrightarrow{0}$, 93. 
While apparently closer to our Monte-Carlo model (the model has no translational noise term) this 'athermal' model still allows transverse displacements controlled by the force term in Eq. (11), at least for continuous pair interactions between the particles, showing that both random noise and transverse component of the forces can induce a macroscopic phase separation. This suggests that the origin and statistical properties of the transverse component of the displacements are not crucial.

Finally, a model of self-propelled hard particles has recently been put forward, which is again reminiscent of our model, in the sense that it does not introduce any energy scale through particle interactions [42]. However, in this model a finite amount of translational noise is introduced as in Eq. (11), suggesting that this model is in fact closer to our generalized Monte-Carlo model where a finite amount of translation noise $\eta$ is introduced.

\section{NONEQUILIBRIUM STRUCTURES AND PHASE DIAGRAM}

We start our investigations with a detailed structural characterization of the stationary phases of the model, exploring in detail the phase diagram $(\phi, \tau)$. The effect of adding a translational noise $\eta>0$ is studied separately in Sec. IV]

\section{A. Phase diagram: Fluid, cluster, and percolated phases}

From a systematic inspection of the steady states obtained for a large range of values of the external parameters $\phi$ and $\tau$, we obtained the phase diagram shown in Fig 3. Typical configurations of the system representative of the different phases are shown in Fig. 1. In the low- $\phi$, low- $\tau$ region, the system sets into a fluid phase with the structure and dynamics of simple liquids at low density, analogous to a dilute suspension of passive disks. This simple phase is expected, as we explicitely constructed the model in order to smoothly recover the equilibrium situation in the limit $\tau \rightarrow 0$.

Increasing the persistence time at constant density induces a strong clustering of the particles (see Fig. 1) and the system enters a phase where finite size clusters characterized by a broad distribution of sizes coexist in space. We call this a cluster phase. We emphasize that this phase is obtained in a genuine stationary state, and that the average cluster size remains finite and independent of the time throughout the cluster phase, with no tendency towards macroscopic phase separation.

Clustering in our model occurs naturally as a result of the competition between self-propulsion (particles move in straight lines) and hard-core repulsion (particles cannot overlap), so that when two particles move persistently towards each other they have to stop when they touch, until decorrelation of the orientation of the displacement

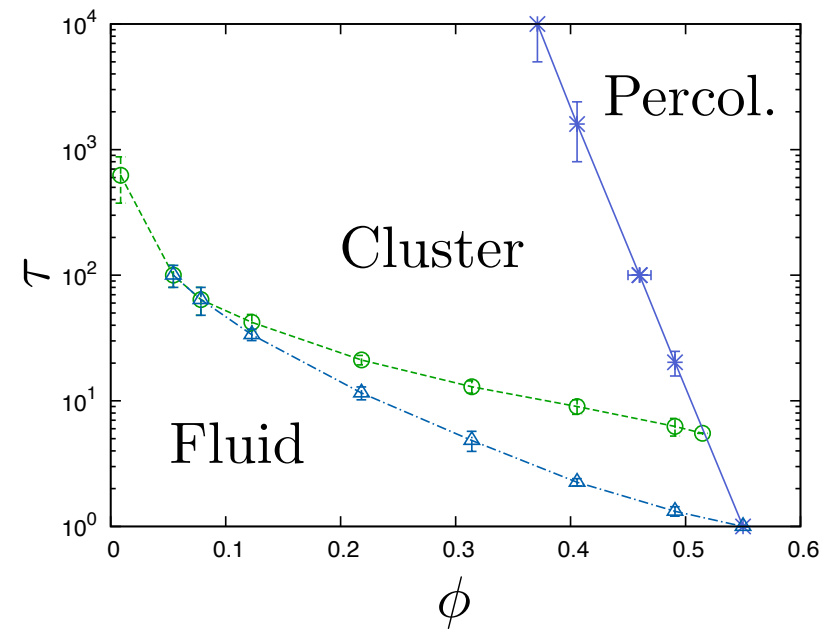

FIG. 3. Phase diagram of the kinetic Monte-Carlo model of self-propelled hard disks in the volume fraction $(\phi)$ and persistence time $(\tau)$ plane. It comprises fluid, cluster and percolated phases. The symbols are numerical estimates of the phase boundaries. While the percolation is uniquely defined, the fluid-cluster boundary is not a phase transition, and we report both a dynamical (circles) and a structural (triangles) criterion to locate this smooth crossover.

vector allows moves that do not create overlaps. Thus, as in other models of active Brownian particles, we expect that self-propulsion is dramatically slowed down in regions where the particle density is large [7, so that active particles have a clear tendency to form aggregates, even in systems where interparticle interactions are purely repulsive.

The formation of clusters in our model is also strongly reminiscent of recent experimental observations in systems of self-propelled colloidal suspensions [17-19], where motility was shown to produce finite size clusters, even at moderate densities.

The two boundaries between the fluid and cluster phases shown in the phase diagram in Fig. 3 were obtained using two different approaches that we describe below. The results show that these two lines differ slightly, which underlies the fact that no genuine phase transition governs the physics of the cluster phase. In other words, the change between fluid and cluster regions in the phase diagram is a smooth crossover.

If the cluster phase obtained at moderate density and large self-propulsion is now compressed at fixed persistence time, the distance between the clusters decreases until a density is reached where the clusters percolate throughout the system. This is the percolated phase in Fig. 3 As discussed below, the separation line between cluster and percolated phases corresponds to a genuine nonequilibrium geometrical transition, whose location can be sharply defined in the thermodynamic limit. In the percolated region, the system has a structure similar to the one found in physical gels, which can be for instance produced in equilibrium conditions in systems 
of attractive colloidal particles.

This analogy strengthens further the idea that selfpropulsion in conjonction with hard-core repulsion induces a kind of effective attractive interaction between active particles [7, 19. However, our results differ from most earlier numerical studies that have reported very little clustering at low and moderate densities, followed by a macroscopic phase separation occurring at intermediate densities and finite self-propulsion. We will analyse below in Sec. IV the possible reasons explaining these differences, and have already alluded many times to the key role played by the translational noise.

\section{B. Characterization of activity-driven cluster phase}

In this subsection, we characterize in detail the structural properties of the cluster phase resulting from the competition between hard core repulsion and selfpropulsion.

The formation of particle aggregates is observed in a wide range of physical situations [44, such as gelation, nucleation, coagulation, polymerisation... Simple models for out-of-equilibrium growth, such as diffusion-limited aggregation (DLA) [45], give rise to structures which can be described in the framework of fractal geometry [46]. Self-propelled disks differ from existing models studied in classic aggregation problems because the basic ingredients are specific to our model. Here, free particles perform a persistent random walk, which is stopped when two or more particles collide, as explained above. In the cluster phase, a steady state is obtained because free particles diffuse and might aggregate to existing clusters, but particles at the surface of these clusters can eventually escape, when their (slowly diffusing) direction of motion points towards the exterior of the clusters. Finite size clusters result from a dynamic equilibrium between aggregation and escape of the self-propelled disks. Such a dynamic process is also observed experimentally in systems of self-propelled colloids.

We have characterized the geometric properties of the obtained clusters in steady state conditions. We define a cluster as a set of disks in contact, that is, with interparticle distance smaller than $\delta_{0}$, the elementary jump length. We then define the cluster mass distribution $P(n)$ as the normalized histogram obtained by measuring the number of clusters containing $n$ particles.

In Fig. 4(a) we show the evolution of $P(n)$ obtained for moderate density, $\phi=0.12$, and increasing the persistence time, using a semi-log representation. The effect of increasing $\tau$ is clear, as the distribution broadens to include larger clusters. Regarding the functional form of the distribution, we find that it is well described by an exponential form for moderate persistence times, but when larger clusters are created, a better functional form is

$$
P(n) \approx n^{-\alpha} \exp \left(-\frac{n}{n^{*}}\right)
$$
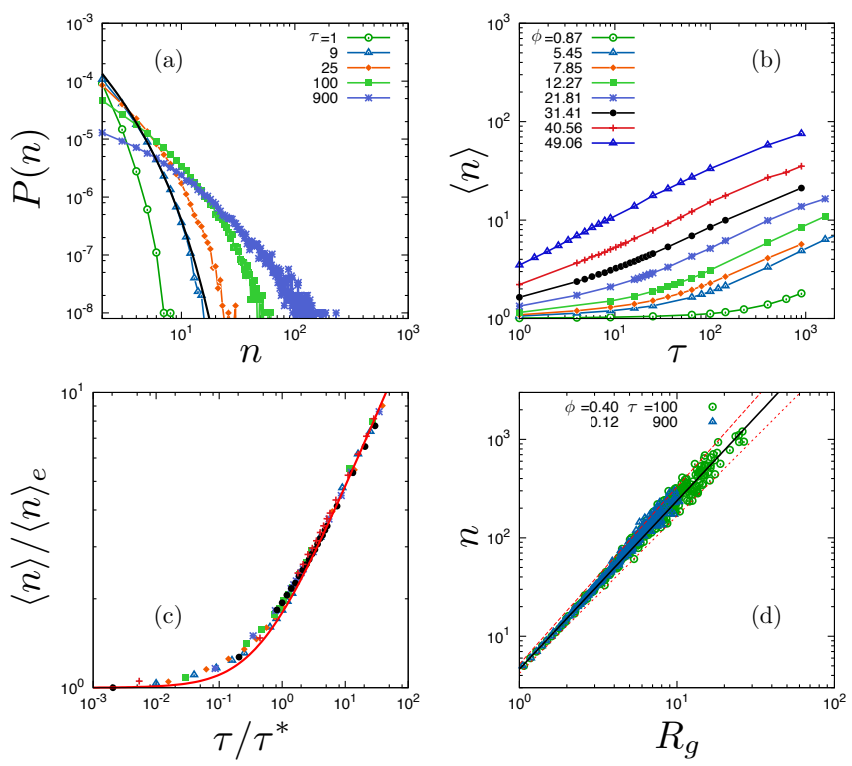

FIG. 4. (a) Cluster size distribution for $\phi=0.12$ and different persistence times. (b) Average cluster size $\langle n\rangle$ as a function of the persistence time $\tau$ for different packing fractions $\phi$. (c) Scaling plot of the average cluster size, rescaled by the equilibrium value $\langle n\rangle_{e}$, while the persistence time is rescaled by the crossover value $\tau^{*}(\phi)$. The solid red line corresponds to the analytic prediction from the kinetic aggregation model, Eq. (27), with $\kappa=3$. (d) Scatter plot of pairs $\left(R_{g}, n\right)$ for clusters obtained at two different state points with measured fractal dimension $d_{R} \approx 1.71$ (full lines). Dashed lines indicate $d_{R}=1.8$ and 1.6, and provide an estimate for the statistical error on the value of $d_{R}$.

where the exponent $\alpha \approx 1.7$, and $n^{*}$ controls the exponential cutoff of the distribution. This functional form, shown in Fig. 4(a) has been found in many instances where clusters are formed [47, 48. It smoothly interpolates between an exponential form when only small clusters are present, and a power law decay, which is found for instance when a percolation of the clusters is obtained. At the percolation transition, the cluster size distribution becomes scale free, $P(n) \sim n^{-\alpha}$. This will be detailed below in Sec. IIID.

As shown in Fig. 4(b), the mean cluster mass defined as

$$
\langle n\rangle=\sum_{n=1}^{\infty} n P(n),
$$

increases for increasing $\tau$ and $\phi$ in the broad range of parameters where steady state properties could be explored numerically. This is in broad agreement with the images shown in Fig. 1.

We can use these measurements to determine a crossover line in the phase diagram delimiting the onset of clustering. In the fluid phase, we find that very few clusters are present, such that $\langle n\rangle$ is a slow-varying function of $\tau$ and $\langle n\rangle \lesssim 2$. However, when the persistence time is increased the average cluster size starts to 
increase more rapidly at a persistence time that depends on the density, because clustering becomes more probable at large density. A simple argument in the spirit of kinetic theory implies that this crossover persistence time should scale as $\tau \sim 1 / \phi$ when $\phi \ll 1$, in good agreement with our numerical data at low density.

The data in Fig. 4(b) suggest the following scaling analysis, that we rationalize below by developing a simple kinetic model for aggregation. For a given packing fraction $\phi$, we measure the average cluster size $\langle n\rangle_{e}$ for passive disks, i.e. at equilibrium. We then normalize $\langle n\rangle$ by $\langle n\rangle_{e}$, and scale the data obtained for different densities using the scaled variable $\tau / \tau^{*}(\phi)$, where $\tau^{*}(\phi)$ is adjusted to produce the best possible collapse. The result of this analysis is shown in Fig. 4(c), which demonstrates that the scaling form

$$
\langle n\rangle=\langle n\rangle_{e} \Phi\left(\frac{\tau}{\tau^{*}}\right)
$$

describes the numerical results very well. We find that $\Phi(x \ll 1) \sim 1$, whereas $\Phi(x \gg 1) \sim x^{1 / 2}$ suggesting that the average cluster size increases as the square root of the persistence time. We rationalize both Eq. (15) and the $\sqrt{\tau}$-dependence in the model described below in Sec. III C.

An interesting outcome of this procedure is a determination, based on structural properties of the system, of a characteristic persistence time $\tau^{*}=\tau^{*}(\phi)$ which marks the onset of clustering. We have reported this crossover line using blue triangular symbols in the phase diagram shown in Fig. 3 .

To analyse further the (possibly fractal) geometry of the clusters, we need to relate the mass of a cluster, $n$, to its size. To this end, we measured the radius of gyration $R_{g}$ of the clusters, defined as

$$
R_{g}^{2}(c)=\frac{1}{n_{c}} \sum_{i \in c}\left|\vec{r}_{i}-\vec{r}_{c}\right|^{2},
$$

where the sum runs over all the particles which belong to a cluster $c$ of mass $n_{c}$ and $\vec{r}_{c}$ denotes the centre of mass of the cluster. We also analysed a different length scale, namely the end-to-end length $\ell$ of the cluster, which is defined as the maximal distance between two particles in the same cluster $c$ :

$$
\ell(c)=\max _{\{i, j\} \in c}\left|\vec{r}_{i}-\vec{r}_{j}\right|
$$

The dependence of the cluster size, $n$, on these geometrical observables allows us to explore their fractal dimension. We define $d_{R}$ by $n \sim R_{g}^{d_{R}}$ and $d_{\ell}$ by $n \sim \ell^{d_{\ell}}$, where $d_{R}$ and $d_{\ell}$ are the fractal dimensions associated with $R_{g}$ and $\ell$ respectively. For fractal clusters, we expect to find $d_{R} \neq d_{\ell}<d(d=2$ being the space dimension), meaning that the mass grows with a characteristic length more slowly than for compact clusters where $d_{R}=d_{\ell}=d$. For instance, for DLA in $d=2$ dimensions, $n \sim R_{g}^{d_{D L A}}$ with $d_{D L A} \approx 1.71$ [45.

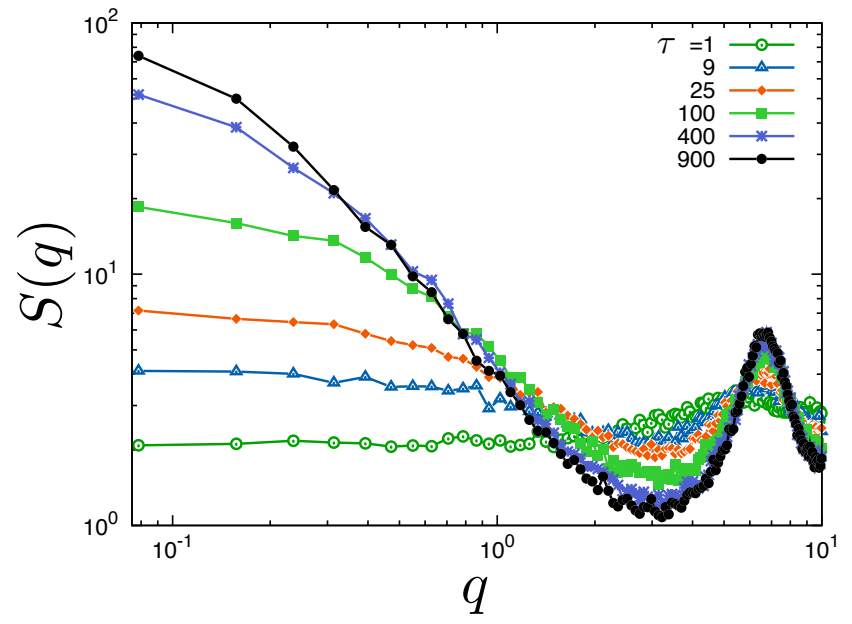

FIG. 5. Static structure factor, Eq. (18), for $\phi=0.12$ and different values of $\tau$. The hard-core repulsion alone (small $\tau$ ) produces a single peak at $q \approx 2 \pi / \sigma$, while increasing clustering enhances the density fluctuations at much lower wavector, i.e. at much larger length scale, revealing the growth of the average cluster size.

From the measure of $R_{g}$ and $\ell$ as a function of the cluster size we extract their fractal dimension. We have checked that finite size effects do not affect these measurements. As illustrated in Fig. 4(d) for two different state points, we obtained the fractal dimensions $d_{R}=1.71 \pm 0.05$ and $d_{\ell}=1.60 \pm 0.05$, independently of both the packing fraction $\phi$ and the persistence time $\tau$ in the cluster phase. These results strongly suggest that clusters in our Monte-Carlo model are always fractal. Moreover, our simulations indicate that, despite the important differences between our model of self-propelled disks and the DLA model, their fractal dimension $d_{R}$ is, within our numerical accuracy, equal to $d_{D L A}$. As explained above, such an agreement is not necessarily expected, as the physics of both aggreation processes is not precisely the same, but this does not seem to affect profoundly the geometry of the clusters.

Finally, we also characterize the structure of the cluster phase using a more standard indicator, namely the static structure factor defined as:

$$
S(q)=\frac{1}{N}\left\langle\left|\sum_{i=1}^{N} e^{i \vec{q} \cdot \vec{r}_{i}}\right|^{2}\right\rangle,
$$

This observable quantifies the strength of density fluctuations on a length scale $\approx 2 \pi /|\boldsymbol{q}|$. In Fig. 5 we show the evolution of the static structure factor $S(q)$ for $\phi=0.12$ as the persistence time is increased. For $\tau=1, S(q)$ has the typical shape obtained for simple fluids at thermal equilibrium, with a maximum at $q \approx 2 \pi / r_{n}$, where $r_{n} \sim \sigma$ is the typical distance between two neighbouring disks. This peak reflects the sole influence of the hardcore repulsion, which is the main ingredient controlling the short-ranged structure of simple liquids. When increasing the persistence time $\tau, S(q)$ strongly increases at 
low- $q$, together with peaks characterizing the local structure at wave vectors multiple of $2 \pi / r_{n}$. This emerging density fluctuations at low- $q$ directly reveal the presence of the clusters observed in real space, see Fig. 1, which produce an inhomogeneous structure over a much larger length scale than in simple fluids. The increase of the intensity of the peaks at larger $q$ reflects the fact that clusters are denser objects than the passive fluid. Our structure factors show strong similarities with the experimental measurements reported in Ref. [17] with the cluster phase of self-propelled colloids.

\section{Kinetic model of reversible aggregation}

We now introduce a simple kinetic model to account for the evolution of the cluster size distribution with the persistence time, using the tools of aggregation models [49].

We denote by $c_{n}(t)$ the number of clusters of mass $n$ at time $t$. By computing the behaviour of $c_{n}(t)$ in the limit $t \rightarrow \infty$, the model should give the main features of the steady-state distribution $P(n)=n c_{n}(t \rightarrow \infty)$ obtained in our Monte-Carlo simulations. Guided by our numerical observations, we make the following assumptions. We assume that the size of a given cluster can only evolve by adding or losing individual particles, as depicted in Fig. 6. Although seemingly reasonable, this assumption implies that we neglect more complicated events such as the aggregation of two clusters, or the breaking of a large cluster into two smaller ones. Therefore, we consider the two following kinetic processes. (1) Aggregation of a single self-propelled disk to an existing cluster of mass $n \geq 1$ with a rate $K_{i n}(n)$. The aggregation rate is estimated by assuming that particles move ballistically at velocity $v_{0}$, so that $K_{\text {in }} \propto \phi v_{0}$. Note that $K_{\text {in }}$ does not depend on the persistence time, see Fig. 6. (2) Evaporation of a selfpropelled disk from a cluster of mass $n>1$ with a rate $K_{\text {out }}(n)$. The rate of evaporation is given by $K_{\text {out }} \propto 1 / \tau$ : it takes about a persistence time $\tau$ to change the orientation of the direction of motion of particles in the boundaries of the cluster in order to escape [22, see Fig. 6. In the following we also assume a mass-independent aggregation and evaporation probability.

In the mean-field approximation neglecting fluctuations, this dynamics can be summarized as

$$
\begin{aligned}
& {[1]+[n] \longrightarrow[n+1] \quad \text { with rate } K_{\text {in }},} \\
& {[n] \longrightarrow[n-1]+[1] \quad \text { with rate } K_{\text {out }},}
\end{aligned}
$$

where $[n]$ denotes a cluster of mass $n$. With these simplifying hypotheses, the time evolution of $c_{n}(t)$ is then ruled by a simple set of differential equations:

$$
\begin{aligned}
\frac{d}{d t} c_{1}(t)= & K_{\text {out }} \sum_{n=2}^{N} c_{n}(t)-K_{\text {in }} c_{1}(t) \sum_{n=1}^{N-1} c_{n}(t), \\
\frac{d}{d t} c_{n}(t)= & K_{\text {in }}\left(c_{1}(t) c_{n-1}(t)-c_{1}(t) c_{n}(t)\right) \\
& +K_{\text {out }}\left(c_{n+1}(t)-c_{n}(t)\right) .
\end{aligned}
$$

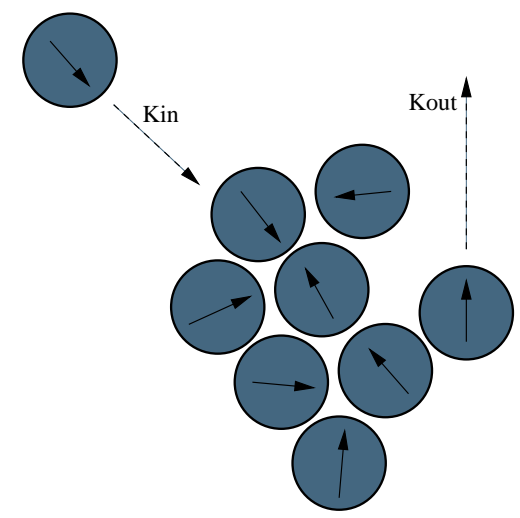

FIG. 6. Schematic representation of the kinetic model for reversible aggregation of self-propelled hard disks. Particles form clusters because excluded volume (represented with blue spheres) opposes self-propulsion (indicated by an arrow). Particles outside the cluster can aggregate with rate $K_{i n}$, while particles at boundaries can escape when the direction of motion has diffused and points toward the exterior of the cluster, which sets the rate $K_{\text {out }} \sim 1 / \tau$.

This is a mean-field model since we are neglecting the spatial structure of the system. The shape and the location of the clusters cannot be described by this approach. A similar modelling has been previously proposed to describe clustering in a suspension of elongated self-propelled rods [5].

In the context of aggregation models, it is common to use an exponential ansatz for the solutions of the kinetic equations 49 . Here we consider solutions of the following form

$$
c_{n}(t)=(1-f(t))^{2} f(t)^{n-1},
$$

where the prefactor $(1-f(t))^{2}$ is found by using the fact that the number of particles in the system is conserved, $\sum_{n=1}^{N} n c_{n}(t)=1, \forall t$. Using the form 23 for $c_{n}$ the kinetic equation 21 becomes:

$$
\frac{d}{d t} f(t)=(1-f(t))^{2}-\lambda f(t), \text { where } \lambda=\frac{K_{\text {out }}}{K_{\text {in }}} .
$$

Its general solution reads

$$
f(t)=\frac{1-\exp \left(-\left(\alpha-\alpha^{-1}\right) t\right)}{\alpha-\alpha^{-1} \exp \left(-\left(\alpha-\alpha^{-1}\right) t\right)}
$$

with $\alpha(\lambda)=1+\frac{\lambda}{2}+\sqrt{\lambda+\frac{\lambda^{2}}{4}}$. The stationary distribution can then easily be obtained by taking the limit $t \rightarrow \infty$ :

$$
\lim _{t \rightarrow \infty} f(t)=\alpha^{-1}, \quad \lim _{t \rightarrow \infty} c_{n}(t) \equiv c_{n}^{s}=\lambda \alpha^{-n} .
$$

Note that the stationarity condition $c_{1}^{s} c_{n}^{s}=\lambda c_{n+1}^{s}$ is automatically verified. As expected, the stationary state distribution is uniquely determined by the relative strength $\lambda=K_{\text {out }} / K_{\text {in }}$ of the two competing processes, 
whose dependence on the persistence time of the selfpropulsion is $\lambda=\kappa / \tau$, which defines the constant $\kappa$, used below as a free parameter.

In order to test the validity of this modelling, we compute the mean cluster size in the stationary state which is given by the second moment of the distribution:

$$
\langle n\rangle=\sum_{n>0} n^{2} c_{n}^{s}=\lambda \alpha^{-1} \frac{1+\alpha^{-1}}{\left(1-\alpha^{-1}\right)^{3}} .
$$

As shown in Fig. 4(c), we find an excellent agreement between this simple kinetic model and the results of the Monte-Carlo simulations of the self-propelled hard disk model, by simply adjusting the constant $\kappa=3$. This good agreement implies that the dependence of the average cluster size is well described by the simple competition between aggregation and evaporation of a single particle as sketched in Fig. 6, and this predicts in particular that the average cluster grows as $\sqrt{\tau}$ when $\tau \gg 1$. Of course, our mean-field model does not provide predictions for the spatial organisation of the clusters, and cannot in particular describe the behaviour of the cluster size distribution when the density increases towards the percolated region.

\section{Percolation transition towards gel-like phase}

We mentioned above that by compressing the cluster phase above a volume fraction of about $\phi \approx 0.4$, a percolation transition emerges, see the phase diagram in Fig. 3 . This transition corresponds to a nonequilibrium version of the contact percolation of hard disks 50 52, because configurational sampling is performed far from equilibrium in the present case. Because the cluster size increases with both $\phi$ and $\tau$, there comes a point where the clusters touch each other and eventually form a systemspanning cluster. This percolation transition is reminiscent of the sol-gel transition observed in colloidal suspensions with attractive interactions.

We use the tools of percolation theory to characterise this transition [50, 53. In Fig. 7]we show the probability $p$ for a disk chosen at random to belong to the largest cluster of the system, measured for $\tau=100$ and various system sizes $N=1000, \cdots, 6000$. In the inset of Fig. 7 , we show the probability $\Pi$ that a disk belongs to a cluster which does not fit into the simulation box, i.e. such that the end-to-end length $\ell>\sqrt{2} L$. These two quantities constitute two alternative methods to determine the location of the percolation transition, as they should jump sharply from 0 to 1 when a percolating cluster emerges in the thermodynamic limit, $N \rightarrow \infty$. As shown in Fig. 7 . both $p$ and $\Pi$ become sharper as system size is increased, although $\Pi$ gives a slightly sharper signature of the percolation in the vicinity of the threshold, which then allows for a more precise determination of its location. From the data shown in Fig. 7, we find a percolation transition at $\phi_{c}(\tau=100)=0.46 \pm 0.01$. In order to construct the

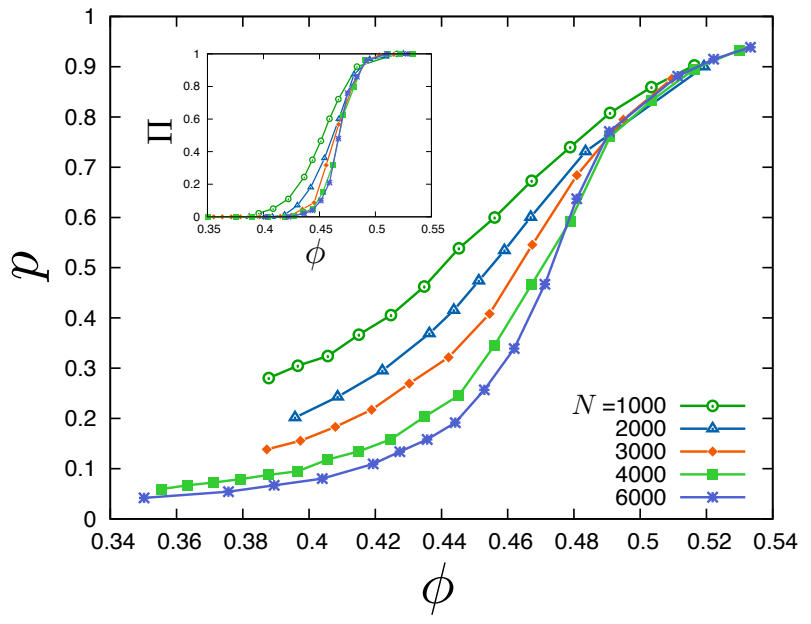

FIG. 7. Probability $p$ that a particle belongs to the largest cluster as a function of $\phi$ for $\tau=100$ and different system sizes. The inset shows the probability $\Pi$ that the largest clusters does not fit into the simulation box as a function of $\phi$ for the same set of parameters. Both probabilities exhibit a sharp jump from 0 to 1 at the percolation transition in the large $N$ limit.

phase diagram we repeated the same analysis for several values of the parameters $\tau$ and $\phi$. These are shown in Fig. 3. Our data are in agreement with the numerical estimation of the contact percolation threshold of passive hard disks $\phi_{c}=0.558 \pm 0.008$ obtained in Ref. [52]. As expected, we find that the percolation density $\phi_{c}(\tau)$ decreases with increasing $\tau$, which mirrors the increase of the cluster size with $\tau$. A similar evolution has been reported in reversible cluster aggregation models by varying the strength of the interaction energy between particles [54, suggesting once more an interesting analogy between the role played by the persistence time in our model, and the role of attractive forces in equilibrium systems.

Another signature of the percolation transition is the algebraic decay of the cluster size distribution, discussed above in the context of the cluster phase. When percolation is approached from the cluster phase, the exponential cutoff of the distribution becomes larger, and exactly at percolation, we find a purely algebraic cluster size distribution $P(n) \sim n^{-\alpha}$ with $\alpha=1.70 \pm 0.05$. We note that this value of $\alpha$ is far from both the value obtained from random bond percolation $(\alpha+1 \approx 2.055)$ [50 and continuum percolation $(\alpha+1 \approx 2.0)$ [55, which indicates that our cluster size distribution at percolation has a different nature from the one found in standard percolation models. We shall see below that the effect of adding noise is to simultaneously make the clusters more compact and to bury the percolation transition inside a phase separated region.

A physically relevant consequence of the percolation transition is the existence of a large area in the $(\phi, \tau)$ phase diagram where the structure of the system is very 
similar to the one of a 'physical gel', characterized by a percolated open structure such as the one shown in Fig. 1 for $\phi=0.49$ and $\tau=900$. It is interesting to note that physical gels are obtained in equilibrium colloidal systems with a careful tuning of interparticle forces, typically using particles with both a strong hard core repulsion and a very short-ranged attraction [47, 56, 57. It is therefore remarkable that similar gel-like structures might spontaneously emerge using purely hard-sphere interactions in conjunction with self-propulsion.

Interestingly, experiments performed with selfpropelled colloidal particles have reported the existence of dense phases with large 'holes', with a structure that is in fact strongly reminiscent of our findings, but this phase has not been characterized in any detail yet 58. We hope our results will motivate further experimental studies of the phase behaviour of self-propelled particles in dense regimes.

\section{EFFECT OF TRANSLATIONAL NOISE: MACROSCOPIC PHASE SEPARATION}

We now study the effect of adding a translational noise of strength $\eta$ in our kinetic Monte-Carlo model, as described in Sec. IIE.

A first important result is the existence of a finite noise strength $\eta_{c}=\eta_{c}(\phi, \tau)>0$ below which the phase diagram obtained for $\eta=0$ remains qualitatively unaffected. This implies that our findings are robust against the addition of a finite amount of translational noise, see Fig. 8. In particular, this means that adding translational noise does not prevent self-propelled disks from aggregating into clusters, nor does the noise necessarily induce a macroscopic phase separation, showing that our results are not 'artefact' of our model where particle displacements are fully slaved to the dynamics of the orientation vector.

However, for $\eta>\eta_{c}$, we find that the system undergoes a macroscopic phase separation reminiscent of liquid-gas demixing (see the rightmost snapshot in Fig. 8). For finite system sizes, we find that the system always reaches a steady state comprising a single dense domain surrounded by a dilute gas of active disks. Some representative snapshots of this situation are shown in Fig. 1 for $\eta=1 / 2$. We checked that this conclusion is robust if the system size is increased, although it takes an increasing simulation time to obtain a single domain in larger systems. We call this region of the three-dimensional phase diagram $(\phi, \tau, \eta)$ the phase-separated region $(\mathrm{PhS})$.

It is not straightforward to determine quantitatively the transition noise $\eta_{c}(\phi, \tau)$ by direct visualisation, see Fig. 8. Therefore we numerically determine $\eta_{c}$ by studying the evolution of the cluster size distribution $P(n)$. For $\eta<\eta_{c}$, the distribution is dominated by small clusters and has an exponentially decaying form. Above $\eta_{c}$ the size distribution is dominated by a macroscopic cluster, so that the shape of $P(n)$ changes dramatically from

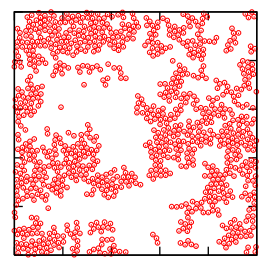

$\eta=0.01<\eta_{c}$

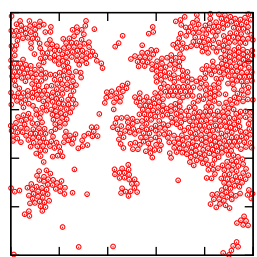

$\eta=0.1 \approx \eta_{c}$

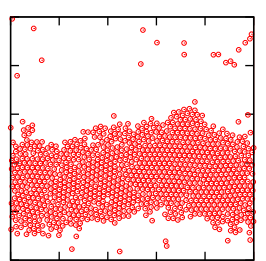

$\eta=0.5>\eta_{c}$
FIG. 8. Snapshots of the steady state obtained for $\phi=0.31$, $\tau=900$ and different noise strength $\eta$. The translational noise favours the formation of more compact clusters, and eventually yields a macroscopic phase separation above a critical value $\eta_{c}=\eta_{c}(\phi, \tau)$.

unimodal to bimodal. We define $\eta_{c}$ by the emergence of a second peak in $P(n)$ at large cluster sizes. From a systematic inspection of $P(n)$ and direct visualisation of the steady state configurations we obtained the value of $\eta_{c}$ for a broad combination of $\phi$ and $\tau$.

In Fig. 9 we show representative phase boundaries $\eta_{c}(\phi, \tau)$ between the phase separated region and the cluster phase for fixed $\tau=900$ over the range $0<\phi<0.4$, Fig. 9(a), and for fixed value of $\phi=0.31$ over the range $1<\tau<2500$, Fig. 9(b). For small persistence times, $\tau \leq 100$, we find that macroscopic phase separation never occurs. When $\tau>100$, we find that $\eta_{c}$ decreases when either $\tau$ or $\phi$ is increased, see Fig. 9. Moreover, we observe that $\eta_{c}$ depends relatively weakly on density, while it is changing quite rapidly with the persistence time $\tau$. Finally, note that when phase separation occurs, it exists down to extremely low densities, in contrast with earlier reports that phase separation only exists at relatively large volume fractions in active Brownian particles [9, 19, 22, 37].

From the snapshots in Fig. 8, one can expect the geometry of the macroscopic cluster in the phase separated region to be different from the one in the cluster phase. We have measured the fractal dimension of the macroscopic cluster at $\eta>\eta_{c}$ for several systems of different size containing up to $N=6000$ disks. We found fractal dimensions $d_{R} \approx d_{\ell} \approx 2$ in the phase separated region. When the system phase separates the resulting macroscopic cluster becomes compact, with $n \sim R_{g}^{2}$. This is illustrated by the snapshots in Figs. 1 and 8 . Interestingly, in the region $\eta<\eta_{c}$, the fractal dimensions $d_{R}$ and $d_{\ell}$ appear to increase continuously from their value at $\eta=0$, $d_{R} \approx 1.7$ and $d_{\ell} \approx 1.6$. For a given $\phi$ and $\tau$, clusters at $\eta=0$ are more ramified than at finite $\eta$. These results are reminiscent of the variation of the fractal dimension of the clusters obtained by reversible cluster aggregation models with finite bond energy 54. Moreover, note that the dense phase at $\eta>\eta_{c}$ appears to be highly ordered, contrarily to the fractal clusters obtained at $\eta=0$ that are characterized by a very disordered structure.

Our central result in this section is that specific combinations of self-propulsion, hard-core repulsion, and a finite amount of translational noise induce a macroscopic 

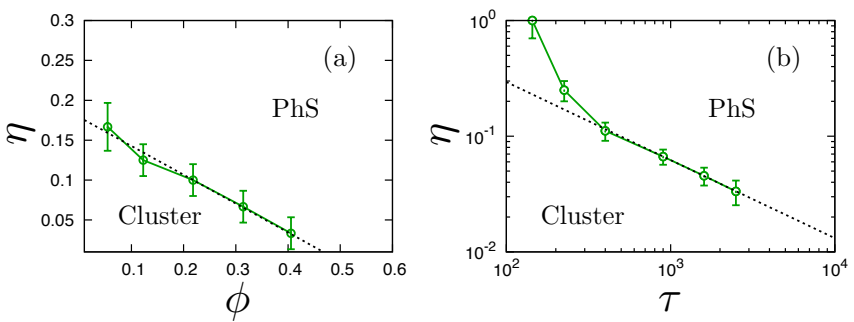

FIG. 9. Phase boundaries between cluster and phase separated phases $(\mathrm{PhS})$ with a finite noise $\eta$. (a) Evolution of $\eta_{c}$ with volume fraction $\phi$ for fixed $\tau=900$ in linear scale showing that a macroscopic phase separation can be obtained down to very low densities. (b) Evolution of $\eta_{c}$ with persistence time $\tau$ for fixed $\phi=0.31$ in log-log scale. No phase separation is found for $\tau \leq 100$. The dotted lines are guides to the eye.

demixing as in equilibrium particle systems with attractive forces. This conclusion is in broad agreement with the phenomenon of motility-induced phase separation that has been thoroughly discussed in the literature [79, 22, 28. Although the interactions between particles are short-ranged and repulsive, the self-propelled disks behave as if there were an effective attraction between them controlled by the persistence time $\tau$. A large amount of work has also been devoted to the kinetics of the phase separation, finding again strong connections with the equilibrium liquid-gas demixing [22, 24, 26].

However, our results shed new light on this nonequilibrium phenomenon. First, we find that translational noise is an essential ingredient to trigger the phase separation. Physically, our interpretation is that the interplay between self-propulsion and hard core repulsion is the primary cause explaining the emergence of clustering: two hard particles moving in persistent motion towards one another cannot cross, and then they have to stop (or at least slow down) when they approach, which results in enhanced clustering.

However, our model with $\eta=0$ shows that this ingredient is not sufficient to trigger phase separation. Indeed, we find that fluctuations of particle displacements with respect to the direction motion imposed by the selfpropulsion is a second crucial ingredient, as it allows internal relaxation inside the clusters. A consequence of these small intra-cluster motions is that clusters become denser and more compact objects. As a result, it becomes more difficult for particles to escape the clusters, while aggregation remains as easy. This argument suggests that the dynamic balance between aggregation and evaporation, which is responsible for the existence of the cluster phase, can be destablized by the addition of noise, to the point that a macroscopic phase separation might occur when aggregation becomes easier than escape. Therefore, we can view the cluster phase in the original model with $\eta=0$ as resulting from a kinetically arrested phase separation.

Another interesting outcome of our study is that phase separation might occur over a broad range of densities and persistence times, including very low volume fractions. Alternative models in the literature devised to study this phase separation typically do not find a macroscopic demixing below $\phi \approx 0.3$, a lower bound which is not present in our approach [37. Overall, these findings suggest that macroscopic phase separation is not necessarily present in self-propelled particle systems, but in fact result from a delicate balance between activity, hardcore repulsion and translational noise. As explained in Sec. IIF previous modelling have indeed studied specific combinations of these three ingredients, by using for instance specific rules for translation/rotation couplings [9, 22. However, our results show that this balance can be tailored with a greater variety than what has been hitherto achieved.

Because the details of the self-propulsion mechanism seem to matter even in the context of very simple models, it should come as no surprise that specific experimental realisations of self-propelled colloidal systems find in some cases a macroscopic phase separation [19, or in some other instances the existence of clustered phases without a phase separation [17, 18].

\section{MICROSCOPIC DYNAMICS OF SELF-PROPELLED DISKS}

In this section, we turn our attention to the microscopic dynamics in the various phases described above, exploring the evolution of dynamic properties as $\phi$ and $\tau$ are varied over a broad range, in the original version of the model, i.e. without translational noise $(\eta=0)$.

\section{A. Nonmonotonic evolution of the diffusion constant}

We have described in Sec. IID the microscopic dynamics of the system of self-propelled hard disks in the dilute limit, $\phi \rightarrow 0$. The behaviour is that of a persistent random walk with a long-time diffusion constant that increases with the persistence time as $D \sim \tau$, so that dynamics becomes faster when $\tau$ increases.

We now investigate the dynamical behaviour at finite density, $\phi>0$, when interparticle interactions play a role. In Fig. 10, we show the evolution of the mean-squared displacement, Eq. (8), for a finite density $\phi=0.12$, and increasing persistence times. As expected for the fluid phase, the behaviour remains purely diffusive, just as in equilibrium. More interesting is the behaviour when $\tau$ becomes larger, since we find a dynamics similar to that of the dilute limit, with a nearly ballistic regime at shorttimes crossing over to diffusive behaviour at long-times that is enhanced with respect to the equilibrium case.

The influence of the interparticle interactions is however very striking. As shown in Fig. 10, we find that particle displacements are initially enhanced by increas- 


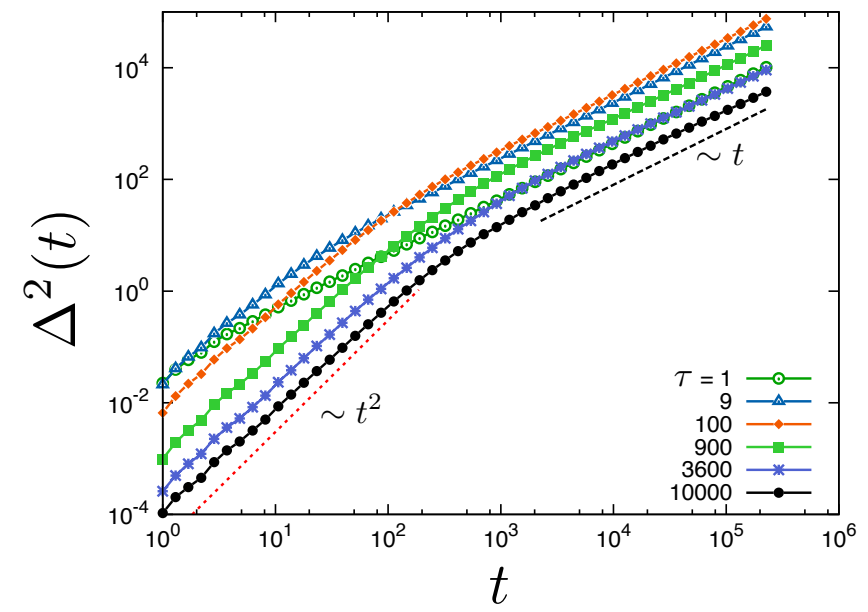

FIG. 10. Mean-squared displacement, Eq. (8), for $\phi=0.12$ and different persistence times $\tau$. Dashed lines indicate ballistic $\left(\Delta^{2} \sim t^{2}\right)$ and diffusive $\left(\Delta^{2} \sim t\right)$ regimes. Note the reentrant behaiour of the dynamics that is initially enhanced by increasing $\tau$ up to $\tau \approx 100$, but slows down when $\tau$ increases further.

ing the persistence time of the self-propulsion, as in the dilute limit. However, a reentrant behaviour is observed when increasing $\tau$ further, and long-time dynamics becomes slower with increasing $\tau$. Therefore, we conclude that increasing self-propulsion might actually slow the dynamics down, which is not a very intuitive result at first sight since it does not happen in the dilute system.

To quantify this effect further, we measure the diffusion constant defined as

$$
D(\phi, \tau)=\lim _{t \rightarrow \infty} \frac{\Delta^{2}(t)}{4 t} .
$$

We present results for the evolution of the diffusion constant with the persistence time at various volume fractions in Fig. 11. The dilute limit behaviour $D \sim \tau$ is recovered for small packing fractions and persistence times. However, for any finite density the diffusion constant exhibits a maximum, and it decreases at large $\tau$, with the asymptotic behaviour given by $D \sim 1 / \tau$ as $\tau \rightarrow \infty$. The maximum of the diffusion constant suggests that there exists an 'optimal' value of the persistence time $\tau_{d}=\tau_{d}(\phi)$ which maximises the diffusivity for a given packing fraction $\phi$. The data in Fig. 11 suggest that $\tau_{d}$ decreases with increasing the density.

The interpretation of the maximum of diffusivity is quite straightforward, because it is a direct consequence of the emergence of the clustered structures described in Sec. III. At small $\tau$ and $\phi$, when small clusters start to form, the spatial structure becomes heterogeneous. This opens large voids where self-propelled particles can move almost freely, i.e. as in the dilute limit. Accordingly, the diffusion constant increases with the persistence time in this regime. However, when $\tau$ increases further, the clusters may become large. The key point is that particles deeply buried inside the clusters can be trapped there for

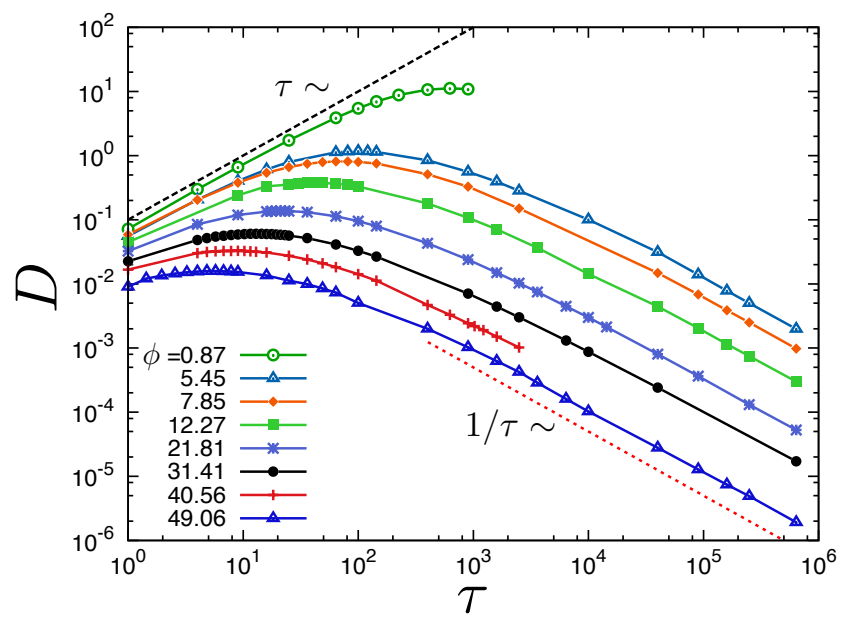

FIG. 11. Diffusion coefficient as a function of the persistence time $\tau$ for different values of the packing fraction $\phi$. The dashed lines describe the behaviour in the dilute limit $D \sim \tau$ which holds when $\phi$ and $\tau$ are small, while the $D \sim 1 / \tau$ describes the large- $\tau$ limit at any finite density, as a consequence of clustering. The crossover between these two regimes defines an optimal persistence time $\tau_{d}(\phi)$ where diffusivity is maximised.

long periods of time. In this regime, increasing $\tau$ can have two opposite effects. Particles in the dilute phase move faster, but particles inside the clusters are arrested for increasing periods of time. Our simulations indicate that when $\tau$ becomes very large, most particles are arrested inside clusters, and the second effect dominates, which explains why $D$ eventually decreases with increasing the self-propulsion. A similar effect has been recently reported for the mobility of active particles driven through a disordered medium [59].

This optimal persistence time $\tau_{d}$ corresponds therefore to a delicate balance between two competing effects: increasing $\tau$ accelerates the dynamics of individual particles, but also produces clustered structures where particles are kinetically trapped. Because clusters form more easily at higher packing fractions, the optimal persistence value $\tau_{d}(\phi)$ decreases when $\phi$ increases.

The optimal value $\tau_{d}$ offers an alternative definition for the location of the crossover between fluid and cluster phases, as it provides a dynamical signature of the emergence of clusters. We report the measured values of $\tau_{d}(\phi)$ obtained from the maximal diffusivity for different packing fractions in the phase diagram in Fig. 3, which gives a crossover line that is in good agreement with the one obtained from directly studying the structure, and already discussed in Sec. IIIB.

The last piece of information we need to discuss in Fig. 11 is the observed large- $\tau$ behaviour of the diffusion constant, namely $D\left(\tau \gg \tau_{d}\right) \sim 1 / \tau$. In this regime, particles alternate between few periods of fast ballistic motion and long periods of kinetic trapping within clusters. As usual, the diffusive dynamics is dominated by the fastest particles, which are the ones sitting on the 
surface of the clusters, which can escape with a rate proportional to $1 / \tau$, as in the sketch of Fig. 6. This limiting rate for cluster escape directly accounts for the scaling of the diffusion constant, $D \sim 1 / \tau$.

\section{B. Non-Fickian diffusion and decoupling}

To further characterise the microscopic dynamics, we compute additional time correlation functions. A natural quantity, which is particularly relevant for scattering experiments, is the self-intermediate scattering function,

$$
F_{s}(q, t)=\frac{1}{N}\left\langle\sum_{i=1}^{N} e^{i \vec{q} \cdot\left[\vec{r}_{i}(t)-\vec{r}_{i}(0)\right]}\right\rangle,
$$

which quantifies particle motion over a typical length scale $\approx 2 \pi / q$. In Fig. 12 , we show the evolution of $F_{s}(q, t)$ from the fluid phase to the cluster phase at fixed packing fraction $\phi=0.12$. As expected for the fluid, the relaxation is fast and exponential in the near-equilibrium case, $\tau=1$. Increasing $\tau$, we observe a first relaxation towards a plateau which emerges at intermediate times (which is not very pronounced), followed by a second slower relaxation. The height of this plateau strongly depends on the value of $\tau$, while its duration does not (and remains relatively short). Increasing $\tau$ further, the dynamics slows down very rapidly, but the time dependence of $F_{s}(q, t)$ does not evolve qualitatively. This behaviour is strongly reminiscent of the viscoelastic relaxation observed in reversible physical gels [47, 60].

Here, the intermediate time plateau emerges as a result of the formation of a heterogeneous structure due to the aggregation of particles. Because both clusters (percolating or not) and a dilute phase coexist, there naturally exist two distinct dynamical families of particles relaxing on two different time scales, and the plateau height reflects their relative weight. Thus, our dynamical study adds one more element to support the physical idea that nonequilibrium persistent motion of repulsive particles produces a physical behaviour reminiscent of equilibrium particles with attractive forces.

The long-time decay of $F_{s}(q, t)$ corresponds to the structural relaxation of the system. From this decay, one can extract a relaxation time scale $t_{r}(q)$, which we define as $F_{s}\left(q, t_{r}(q)\right)=0.2$ (the precise value 0.2 is irrelevant). It is interesting to study the wave vector dependence of the relaxation time, as displayed in Fig. 12 . Whereas purely diffusive behaviour, $t_{r} \sim q^{-2}$, is observed for near-equilibrium dynamics at small $\tau$, the structural relaxation time displays a crossover between two different regimes when $\tau$ increases. Diffusive behaviour is still observed but only when $q$ is very low, whereas the behaviour at larger $q$ is very strongly non-Fickian with $t_{r}(q)$ being only weakly dependent on wave vector.

Deviations from Fickian behaviour are generically expected in systems exhibiting strong dynamic heterogeneity 61. In particular, systems characterized by the exis-
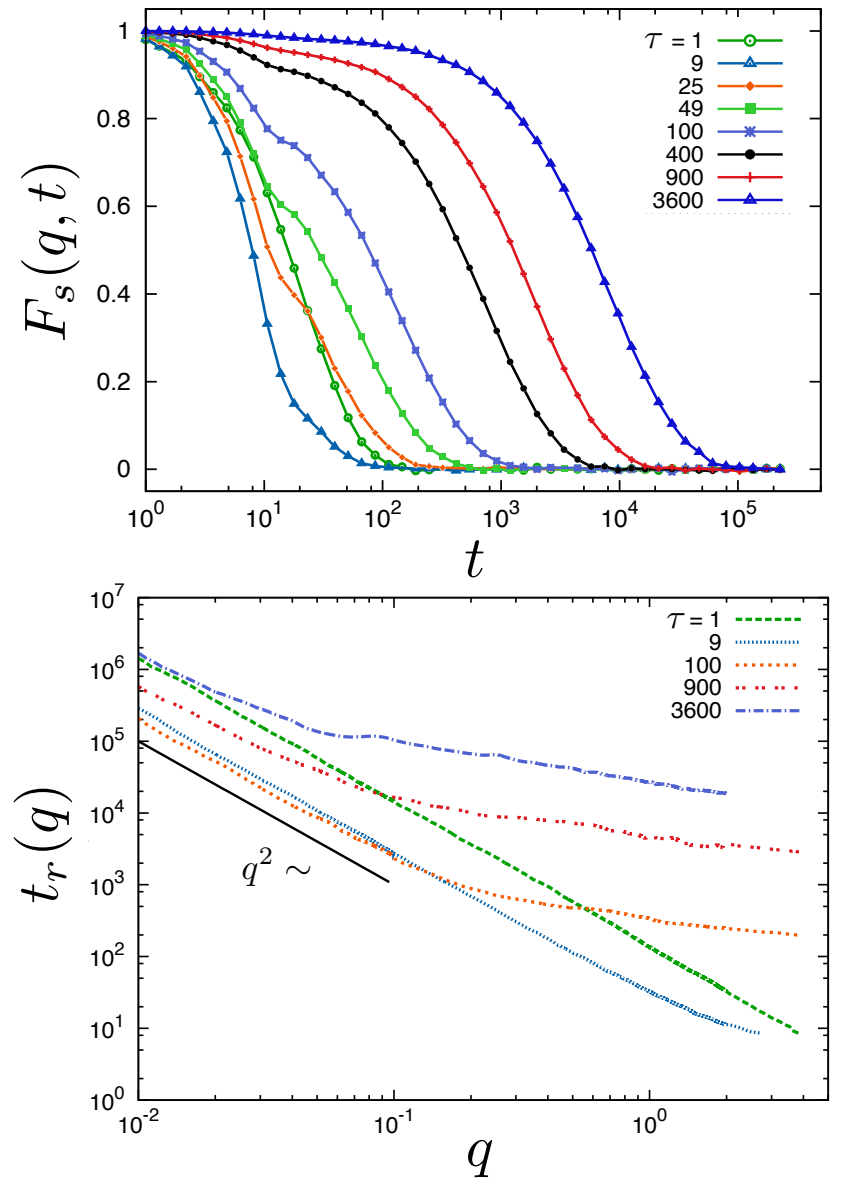

FIG. 12. Top: Self-intermediate scattering function, Eq. 29 for $q=1.20$ for $\phi=0.12$ and different values of the persistence time. Bottom: Relaxation time $t_{r}(q)$ for the same density, with Fickian behaviour $t_{r} \sim 1 / q^{2}$ indicated with a dashed line.

tence of distinct dynamic populations (e.g. fast and slow particles) with kinetic exchanges between the two populations 60, 62 all exhibit two-step decay in intermediate scattering functions and non-Fickian dynamics at short scale of the type shown in Fig. 12 .

The $q$-dependence of the relaxation is easily explained in this dynamically heterogeneous scenario. In a system with fast and slow particles, it is the slow population which controls the long-time decay of the selfintermediate scattering function at large $q$, which basically quantifies how long it takes for initially trapped particles to start moving. On the other hand, the low- $q$ behaviour is controlled by the long-time diffusion constant, $D$, which does not necessarily quantify the same dynamic process. In the two-population scenario, for instance, $D$ is essentially controlled by the exchange rate between the two families, whereas $t_{r}(q)$ at large $q$ is controlled by the relaxation time of the slow one [63]. These two distinct measures of the relaxation time only become equivalent when dynamics is homogeneous and purely Fickian, in which case one has $t_{r}(q) \sim 1 /\left(D q^{2}\right)$. Our re- 
sults suggest instead that $t_{r}$ increases much faster than $1 / D$ with increasing $\tau$. The data in Fig. 12 suggest for instance a growth of about 3 orders of magnitude of the product $D \times t_{r}(q)$ (for $\phi=0.12$ and $q=1.2$ ) and $\tau$ increasing by about 4 orders of magnitude. The independent evolution of $t_{r}(q)$ and $1 / D$ is called a 'decoupling' phenomenon in the context of dynamically heterogeneous materials 61, 64.

Non-Fickian diffusion and decoupling phenomena have been reported in a large number of physical systems 61, 64, from supercooled liquids approaching a glass transition to dense granular, colloidal suspensions, and colloidal gels [60, 65]. For the latter type of systems, dynamic heterogeneity is a direct (and physically unsurprising) consequence of a heterogeneous structure 60, 66, as is the case for the present model where the structure in cluster and percolated phases is directly responsible for the peculiar dynamic features reported in this section.

\section{Dynamic heterogeneity}

The decoupling of the evolution of the diffusion constant and the relaxation time together with the strongly non-Fickian wave vector dependence discussed above suggest that microscopic dynamics is spatially heterogeneous in our model of self-propelled hard disks.

A simple way to observe this dynamic heterogeneity more directly is to focus on distributions of particle displacements. We have measured the self-part of the van Hove function, defined as

$$
G_{s}(x, t)=\frac{1}{N}\left\langle\sum_{i=1}^{N} \delta\left(x-\left|x_{i}(t)-x_{i}(0)\right|\right)\right\rangle .
$$

Because the system is isotropic, we can average the above function along both space directions. This function is akin to a (normalized) histogram of single particle displacements measured over a fixed time delay, which takes a simple Gaussian form for purely Fickian dynamics. Note that $G_{s}(x, t)$ is also related to $F_{s}(q, t)$ by a Fourier transform.

In Fig. 13 , we show the time evolution of $G_{s}(x, t)$ for $\phi=0.12$ and two values of the persistence time, $\tau=100$ and $\tau=900$. To better appreciate the time evolution of the shape of these distributions, it is convenient to use a rescaled unit $x / \sqrt{t}$, as dictated by the long-time diffusive limit. While the distributions eventually converge to the expected Gaussian distribution, quite strong deviations from Gaussian behaviour are observed at finite times. In particular, the coexistence of nearly arrested and fastmoving particles is obvious at short times, since the van Hove function displays both a very narrow peak at the origin stemming from immobile particles, and broad tails (i.e. broader than the corresponding Gaussian distribution) stemming from fast particles. The distinction between mobile and immobile particles becomes more pronounced as the persistent time is increased. Such broad
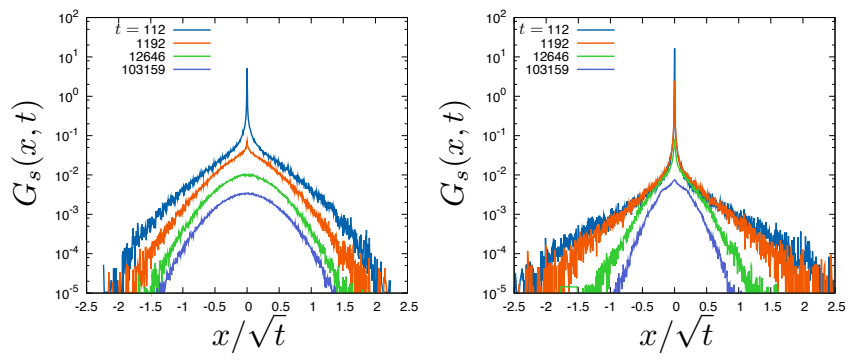

FIG. 13. Distribution of particle displacements, Eq. (30), for $\phi=0.12$, and $\tau=100$ (left) and $\tau=900$ (right). The distributions are composed of a superposition of immobile (near $x \approx 0$ ) and very mobile (in the nearly exponential tails) particles, and converge to Gaussian form at long times.

tails are observed in a large number of dynamically heterogeneous materials [61, where they are usually found to be described by an exponential decay [67, as is also found in the data shown in Fig. 13, especially at large $\tau$.

As time increases, the height of the central peak decreases because the population of immobile particles naturally decreases with time (eventually at long times all particles become mobile). As expected, this arrested structure stays immobile over a longer period when $\tau$ increases. When all particles have finally moved, the tails of the distribution become closer and closer to the corresponding Gaussian distribution, and dynamic heterogeneity is washed out.

These distributions confirm that the microscopic dynamics is heterogeneous due to the coexistence of arrested particles inside clustered regions, and free particles diffusing rapidly in dilute regions. This accounts for both non-Fickian diffusion and decoupling of diffusion constant and structural relaxation time. This physical behaviour is reminiscent of the dynamic heterogeneity discussed in the context of colloidal gels 60, 66, 68. It seems clear that the simple kinetic models developed in the context of colloidal gels to describe analytically the shape of the van Hove distributions [60, 62] would be directly applicable to the present model. We suggest that such models would also be useful in the context of experimental investigations of dynamic heterogeneity in selfpropelled particle systems, but this line of investigation has been little explored.

\section{SUMMARY AND CONCLUSION}

To summarize, we have introduced and studied in great detail a kinetic Monte-Carlo model for self-propelled hard disks, which contains a minimal amount of free parameters. As a result, the model is quite crude, but it still captures essential features of the competition between thermal fluctuations, self-propulsion and hard core repulsion between the disks. Although seemingly simpler than alternative models in the literature, we have demonstrated that it is also somewhat more flexible, in the sense that 
it allows us to disentangle more clearly the specific roles played by each ingredient in the model, but also to study combinations of parameters that have not been explored in earlier models.

Our main findings are the emergence of non-trivial nonequilibrium structures at finite density, which take the form of a cluster phase at moderate density, eventually percolating into a gel-like phase at larger density. Adding a controlled amount of translational noise to the model allows us to connect our results to earlier studies of a motility-induced macroscopic phase separation.

We have compared our results to experimental model systems of self-propelled colloidal suspensions for which both a cluster phase and a phase separated state have been observed as well 17-19. We note that the gel-like phase we obtain at large density has not been studied experimentally in any detail yet. Our investigations of the microscopic dynamics of this phase suggest that it is potentially a very interesting regime, characterized in particular by strong dynamic heterogeneities, similar to the ones found in colloidal gels formed with attractive particles.

In future work, we aim at investigating more closely the striking analogies underlined in this work between the nonequilibrium physics of self-propelled disks and the equilibrium behaviour of systems with short-range attractive forces. Additionally, we believe the present kinetic Monte-Carlo model is particularly well-suited to study the effect of self-propulsion at large densities, since it captures the essential elements of the competition between slow dynamics emerging because of crowding at large density, and the activity of the particles that provides the fuel needed to move them faster. While few numerical [36, 37, 42 and theoretical [69, 70, studies of this competition have appeared, we also encourage experimental work in that direction.

\section{ACKNOWLEDGMENTS}

We thank T. Kawasaki for useful exchanges. The research leading to these results has received funding from the European Research Council under the European Union's Seventh Framework Programme (FP7/20072013) / ERC Grant agreement No 306845.
[1] S. Ramaswamy, Annual Review of Condensed Matter Physics 1, 323 (2010).

[2] T. Vicsek and A. Zafeiris, Physics Reports 517, 71 (2012).

[3] M. C. Marchetti, J. F. Joanny, S. Ramaswamy, T. B. Liverpool, J. Prost, M. Rao, and R. A. Simha, Reviews of Modern Physics 85, 1143 (2013).

[4] J. Toner, Y. Tu, and S. Ramaswamy, Annals of Physics 318, 170 (2005).

[5] F. Peruani, A. Deutsch, and M. Bär, Physical Review E 74, 030904 (2006).

[6] F. Ginelli, F. Peruani, M. Bär, and H. Chaté, Physical Review Letters 104, 184502 (2010).

[7] J. Tailleur and M. Cates, Physical Review Letters 108, 218103 (2008).

[8] M. E. Cates, Reports on Progress in Physics 75, 042601 (2012).

[9] Y. Fily and M. Marchetti, Physical Review Letters 108, 235702 (2012).

[10] A. Bricard, J.-B. Caussin, N. Desreumaux, O. Dauchot, and D. Bartolo, Nature 503, 95 (2013).

[11] V. Narayan, S. Ramaswamy, and N. Menon, Science 317, 105 (2007).

[12] J. Deseigne, O. Dauchot, and H. Chaté, Physical Review Letters 105, 098001 (2010).

[13] W. F. Paxton, K. C. Kistler, C. C. Olmeda, A. Sen, S. K. St. Angelo, Y. Cao, T. E. Mallouk, P. E. Lammert, and V. H. Crespi, Journal of the American Chemical Society 126, 13424 (2004).

[14] L. Hong, S. Jiang, and S. Granick, Langmuir 22, 9495 (2006).

[15] L. Hong, A. Cacciuto, E. Luijten, and S. Granick, Nano Letters 6, 2510 (2006).
[16] G. Volpe, I. Buttinoni, D. Vogt, H.-J. Kümmerer, and C. Bechinger, Soft Matter 7, 8810 (2011).

[17] I. Theurkauff, C. Cottin-Bizonne, and J. Palacci, Physical Review Letters 108, 268303 (2012).

[18] J. Palacci, S. Sacanna, A. P. Steinberg, D. J. Pine, and P. M. Chaikin, Science 339, 936 (2013).

[19] I. Buttinoni, J. Bialké, F. Kümmel, H. Löwen, C. Bechinger, and T. Speck, Physical Review Letters 110, 238301 (2013).

[20] H. P. Zhang, A. Be'er, E.-L. Florin, and H. L. Swinney, Proceedings of the National Academy of Sciences of the United States of America 107, 13626 (2010).

[21] H. Chaté, F. Ginelli, and R. Montagne, Physical Review Letters 96, 180602 (2006).

[22] G. Redner, M. Hagan, and A. Baskaran, Physical Review Letters 110, 055701 (2013).

[23] A. Thompson and J. Tailleur, p. 02029 (2011).

[24] J. Bialké, H. Löwen, and T. Speck, EPL (Europhysics Letters) 103, 30008 (2013).

[25] M. Cates and J. Tailleur, EPL (Europhysics Letters) 101, 20010 (2013).

[26] J. Stenhammar, A. Tiribocchi, R. J. Allen, D. Marenduzzo, and M. E. Cates, Physical Review Letters 111, 145702 (2013).

[27] J. Stenhammar, D. Marenduzzo, R. J. Allen, and M. E. Cates, Soft Matter 10, 1489 (2014).

[28] R. Wittkowski, A. Tiribocchi, J. Stenhammar, R. J. Allen, D. Marenduzzo, and M. E. Cates, arXiv:1311.1256 (2013).

[29] J.-P. Hansen and I. R. McDonald, Theory of simple liquids (Elsevier, 1990).

[30] D. Loi, S. Mossa, and L. F. Cugliandolo, Physical Review E 77, 051111 (2008). 
[31] J. Bialké, T. Speck, and H. Löwen, Physical Review Letters 108, 168301 (2012).

[32] M. E. Newman and G. T. Barkema, Monte Carlo methods in statistical physics (Oxford University Press, 1999).

[33] D. P. Landau and K. Binder, A guide to Monte Carlo simulations in statistical physics (Cambridge University Press, 2009).

[34] I. Gotze and G. Gompper, Physical Review E 82, 041921 (2010).

[35] J. Taktikos, V. Zaburdaev, and H. Stark, Physical Review E 85, 051901 (2012).

[36] L. Berthier, arxiv:1307.0704 (2013).

[37] Y. Fily, S. Henkes, and M. C. Marchetti, Soft Matter 10, 2132 (2014).

[38] A. Wysocki, R. G. Winkler, and G. Gompper, arXiv:1308.6423 (2013).

[39] B. Mognetti, A. Šarić, S. Angioletti-Uberti, A. Cacciuto, C. Valeriani, and D. Frenkel, Physical Review Letters 111, 245702 (2013).

[40] P. Ball, Physics 6, 134 (2013).

[41] J. Stenhammar, A. Tiribocchi, R. J. Allen, D. Marenduzzo, and M. E. Cates, Physical Review Letters 111, 145702 (2013).

[42] R. Ni, M. Stuart, and M. Dijkstra, Nature Communications 4, 2704 (2013).

[43] L. Berthier and W. Kob, Journal of Physics: Condensed Matter 19, 205130 (2007).

[44] P. Meakin, Fractals, scaling and growth far from equilibrium (Cambridge University Press, 1998).

[45] T. A. Witten and L. M. Sander, Physical Review Letters 47, 1400 (1981).

[46] P. Meakin, in Phase Transitions and Critical Phenomena vol 12, edited by C. Domb and J. L. Lebowitz (Academic Press, 1988).

[47] E. Zaccarelli, Journal of Physics: Condensed Matter 19, 323101 (2007).

[48] R. Soto and R. Golestanian, Physical Review E 89, 012706 (2014).

[49] P. Krapivsky, S. Redner, and E. Ben-Naim, A kinetic view of statistical physics (Cambridge University Press, 2010).

[50] D. Stauffer and A. Aharony, Introduction to Percolation Theory (Taylor \& Francis, 1991).
[51] K. W. Kratky, Journal of Statistical Physics 52, 1413 (1988).

[52] T. Shen, C. S. O'Hern, and M. D. Shattuck, Physical Review E 85, 011308 (2012), ISSN 1539-3755.

[53] D. Stauffer, A. Coniglio, and M. Adam, Advances in Polymer Science 44, 103 (1982).

[54] J. Jin, K. Parbhakar, L. Dao, and K. Lee, Physical Review E 54, 997 (1996).

[55] E. T. Gawlinski and H. E. Stanley, Journal of Physics A: Mathematical and General 14, L291 (1981).

[56] P. J. Lu, J. C. Conrad, H. M. Wyss, A. B. Schofield, and D. A. Weitz, Physical Review Letters 96, 028306 (2006).

[57] P. Lu, E. Zaccarelli, F. Ciulla, A. Schofield, F. Sciortino, and D. Weitz, Nature 453, 499 (2008).

[58] I. Theurkauff, Ph.D. thesis, Université Lyon 1 (2013).

[59] C. Reichhardt and C. Reichhardt, arXiv:1402.3260 (2014).

[60] P. I. Hurtado, L. Berthier, and W. Kob, Physical Review Letters 98, 135503 (2007).

[61] L. Berthier, G. Biroli, P. Bouchaud, and R. L. Jack, in Dynamical Heterogeneities in Glasses, Colloids and Granular Media, edited by L. Berthier, G. Biroli, J. Bouchaud, L. Cipelletti, and W. van Saarloos (Oxford University Press, 2011).

[62] P. Chaudhuri, Y. Gao, L. Berthier, M. Kilfoil, and W. Kob, Journal of Physisics: Condensed Matter 20, 244126 (2008).

[63] L. Berthier, D. Chandler, and J. P. Garrahan, EPL (Europhysics Letters) 69, 320 (2005).

[64] M. Ediger, Annual review of physical chemistry pp. 99128 (2000).

[65] L. Guo and E. Luijten, Journal of Polymer Science Part B: Polymer Physics 43, 959 (2005).

[66] C. J. Dibble, M. Kogan, and M. J. Solomon, Physical Review E 74, 041403 (2006).

[67] P. Chaudhuri, L. Berthier, and W. Kob, Physical Review Letters 99, 060604 (2007).

[68] Y. Gao and M. Kilfoil, Physical Review Letters 99, 078301 (2007).

[69] L. Berthier and J. Kurchan, Nature Physics 5, 310 (2013).

[70] T. Farage and J. Brader, arXiv:1403.0928 (2014). 